\title{
Cartografía geológica para la gestión del geopatrimonio y la planeación de rutas geoturísticas: Aplicación en el Geoparque Mundial de la UNESCO Comarca Minera, Hidalgo
}

\section{Geological mapping as a tool for the management of geoheritage and geotourism: case study of the Comarca Minera, Hidalgo UNESCO Global Geopark}

\author{
Carles Canet*a, Juan Carlos Mora-Chaparro ${ }^{\mathrm{a}}$, Arturo Iglesias ${ }^{\mathrm{a}}$, Miguel A. Cruz-Pérez ${ }^{\mathrm{a}}$, Erika Salgado-Martínez ${ }^{\mathrm{b}}$, \\ David Zamudio-Ángeles ${ }^{\mathrm{a}}$, Elisa Fitz-Díaz ${ }^{\mathrm{c}}$, Raymundo G. Martínez-Serrano ${ }^{\mathrm{a}}$, Alondra Gil-Ríos ${ }^{\mathrm{a}}$, Joan Poch $^{\mathrm{d}}$ \\ ${ }^{a}$ Instituto de Geofísica, Universidad Nacional Autónoma de México, Del. Coyoacán, 04510, Ciudad de México \\ ${ }^{b}$ Coordinación de Vinculación Institucional, Universidad Nacional Autónoma de México, Del. Coyoacán, 04510, Ciudad de México \\ ${ }^{c}$ Instituto de Geología, Universidad Nacional Autónoma de México, Del. Coyoacán, 04510, Ciudad de México \\ ${ }^{d}$ Departament de Geologia, Universitat Autònoma de Barcelona, 08193, Barcelona, España
}

\section{Resumen}

La Comarca Minera de Hidalgo (México) es desde mayo de 2017 un Geoparque Mundial de la UNESCO; dicha denominación se justifica por un geopatrimonio de relevancia internacional que incluye: (a) el sistema epitermal (Ag- $\mathrm{Au}$ ) de clase mundial de Pachuca-Real del Monte; (b) la localidad tipo de la tridimita y la cristobalita; (c) los basaltos con disyunción columnar de Huasca de Ocampo (conocidos como Prismas Basálticos de Santa María Regla); y (d) el patrimonio industrial minero, que comprende vestigios prehispánicos y haciendas de beneficio coloniales. El geoparque se articula alrededor de una red de 31 geositios cuyo propósito general es la geoconservación y el aprovechamiento turístico y educativo del geopatrimonio. El mapa geoturístico del geoparque, presentado en esta contribución, se ha elaborado con el fin de que sea usado como un recurso educativo y, además, como una herramienta para la catalogación, aprovechamiento y conservación de la geodiversidad.

Palabras clave: Mapa geológico; Geodiversidad; Geopatrimonio; Geoturismo; Geoconservación; Enseñanza de las Ciencias de la Tierra.

\begin{abstract}
The Comarca Minera, Hidalgo UNESCO Global Geopark (Mexico) was formally designated on May 2017; this label is justified by an outstanding geoheritage that includes: (a) the Pachuca-Real del Monte world class epithermal deposits (Ag-Au); (b) the type locality for two minerals: tridymite and cristobalite; (c) the Huasca de Ocampo columnar basalts, first described by Alexander von Humboldt in 1803; and (d) a broad mining heritage that includes remains of pre-Columbian obsidian industry and New Spain mining haciendas (i.e. colonial metallurgy plants). This geopark encompasses a network of 31 geosites, whose general purpose is the educational and touristic use of geoheritage. The geotouristic map of the recently declared geopark, here presented, was conceived as an educational resource, as well as a tool for the touristic development and for the categorization, study and protection of geodiversity.
\end{abstract}

Keywords: Geologic map; Geodiversity; Geoheritage; Geotourism; Geoconservation; Earth Science Education.

\footnotetext{
(C) Carles Canet, Juan Carlos Mora-Chaparro, Arturo Iglesias, Miguel A. Cruz-Pérez, Erika Salgado-Martínez, David Zamudio-Ángeles, Elisa Fitz-Díaz, Raymundo G. Martínez-Serrano, Alondra Gil-Ríos, Joan Poch. Published by Terra Digitalis.

This is an Open Access article distributed under the terms of the Creative Commons Attribution License (https://creativecommons.org/licenses/by-nc-sa/4.0/),
}

which permits non-commencial sharing of the work and adaptions, provided the original work is properly cited and the new creations are licensed under identical terms.

*E-mail address: ccanet@geof isica.unam.mx 


\section{Introducción}

Los mapas geológicos son representaciones cartográficas de la disposición sobre la superficie del terreno de las distintas unidades rocosas que afloran en un área concreta, las cuales se definen por sus características litológicas y edad, que permiten apreciar las relaciones geométricas y temporales entre ellas; además, comúnmente muestran la distribución de diversos rasgos geológicos como pliegues, fallas, lineamientos, foliación, así como la orientación — rumbo y buzamiento- de estructuras y planos de estratificación (e.g. IGME, 2016; CGS, 2016). Los mapas geológicos tienen una gran variedad de usos y propósitos que incluyen la exploración de recursos minerales e hídricos, estudios de riesgos geológicos, geología ambiental e ingeniería del terreno, así como investigación científica en todas las áreas de las Ciencias de la Tierra (e.g. Soller, 2004; CGS, 2016). Además, la cartografía geológica, como abstracción espacio-temporal de las rocas, es en sí un recurso didáctico esencial para la formación de los geocientíficos (e.g. Baker et al., 2012), y los mapas geológicos constituyen una herramienta útil para la ordenación del territorio (e.g. Greiving et al., 2016).

La geodiversidad, definida como la diversidad natural de características y elementos geológicos (rocas, minerales, fósiles), geomorfológicos (formas de relieve, procesos físicos) y de los suelos (Gray, 2004), es asimismo un recurso geológico, pues condiciona la distribución de la biodiversidad, de los paisajes y de los usos del suelo (Prosser et al., 2010), y además puede generar un beneficio económico directo a través del geoturismo (Hose, 1995; 2016; Hose y Vasiljević, 2012). Como recurso geológico que es, la geodiversidad se apoya en la cartografía geológica para su catalogación y valoración sistemáticas, así como para el estudio de su potencial aprovechamiento geoturístico. Al conjunto de elementos geológicos de una región determinada que son catalogados y a los que se asigna un valor por su interés científico, cultural y/o educativo se le denomina geopatrimonio (Carcavilla et al., 2007). Los puntos de interés geológico son inventariados como geositios, de acuerdo con la iniciativa Geosites promovida por la IUGS desde 1996 (Palacio-Prieto, 2013a; IUGS-GTG, 2016).

Los Geoparques Mundiales de la UNESCO (geoparques, de aquí en adelante) son territorios con un geopatrimonio sobresaliente (i.e. que incluye geositios de importancia internacional) que es utilizado para promover el desarrollo sustentable mediante el geoturismo, así como la educación, integrando actividades tradicionales y la cultura local (GGN, 2017; UNESCO Global Geoparks, 2017). Actualmente la mayoría de geoparques se hallan en Europa y Asia, habiendo únicamente seis en toda América (dos en Canadá, dos en México, uno en Uruguay y uno en Brasil; UNESCO Global Geoparks, 2017). México es un país con una enorme diversidad geológica y paisajística (Palacio-Prieto, 2013a y b) que cuenta, desde el 5 de mayo de 2017, con dos geoparques formalmente reconocidos (UNESCO Global Geoparks, 2017): (a) Comarca Minera, Hidalgo, y (b) Mixteca Alta, Oaxaca.

En numerosos geoparques los mapas geológicos se utilizan como un recurso educativo y, además, son la base para la catalogación y aprovechamiento de los geositios (e.g. geoparques de Stonehammer y Tumbler Ridge, Canadá; Katla, Islandia; Ore of the Alps, Austria; Hong Kong Geopark, China). Asimismo, en numerosos países los mapas geológicos son utilizados para: (a) el ordenamiento de los territorios ricos en geopatrimonio, aún de aquellos que no cuentan con una designación oficial como geoparque (e.g. SGU, 2016); y (b) la realización de inventarios de geositios en general, tarea que suelen realizar los servicios geológicos (e.g. IELIG, 2016).

Fundamentado en un patrimonio geológico sobresaliente, el cual tiene una notable influencia en el entorno biótico y cultural, así como en una estrategia para el desarrollo sustentable basada en el geoturismo, el Geoparque Comarca Minera (GCM) busca tener un impacto positivo en la sociedad hidalguense mediante programas educativos y de divulgación de la ciencia, y consolidarse como Geoparque Mundial de la UNESCO. La cartografía geológica ha sido una herramienta fundamental para establecer el inventario de geositios del GCM; esta labor y el mapa geológico del territorio geoparque (ver Lámina 1) se describen en la presente contribución.

\section{El geoparque Comarca Minera, Hidalgo: Descripción general y geopatrimonio}

Con un área de $1848 \mathrm{~km}^{2}$, el GCM se localiza en la parte centro-sur del estado de Hidalgo y comprende nueve municipios (enteros): (a) Atotonilco el Grande, $427 \mathrm{~km}^{2}$; (b) Singuilucan, $334 \mathrm{~km}^{2}$; (c) Huasca de Ocampo, $306 \mathrm{~km}^{2}$; (d) Pachuca de Soto, $195 \mathrm{~km}^{2}$; (e) Epazoyucan, $175 \mathrm{~km}^{2}$; (f) Mineral del Chico, 118 km²; (g) Omitlán de Juárez, 110 km²; (h) Mineral de la Reforma, $106 \mathrm{~km}^{2}$; (i) Mineral del Monte, $77 \mathrm{~km}^{2}$. La población total del territorio del GCM es aproximadamente de 500,000 habitantes, distribuidos en 454 núcleos de población (INEGI, 2013). La zona goza de una red de vías de comunicación terrestre, con carreteras pavimentadas que enlazan las cabeceras municipales y muchas poblaciones menores.

\subsection{Marco fisiográfico y geológico}

El elemento fisiográfico más relevante del GCM lo constituyen las sierras de Pachuca y de Las Navajas, con cotas máximas en la Peña de las Ventanas (3090 msnm) y en el Cerro de las Navajas (3212 msnm), respectivamente (INEGI, 2007). Este conjunto orográfico tiene una orientación general NW-SE y separa (a) el área de la Sierra Baja y Barranca de Metztitlán, con altitudes de $\sim 1300-2000 \mathrm{msnm}$, al noreste, de (b) el extremo septentrional de la Cuenca de México, con una altitud de $\sim 2200$ - $2400 \mathrm{msnm}$, al suroeste.

El GCM se localiza entre dos provincias fisiográficas (cf. Raisz, 1959): (a) la Faja Volcánica Transmexicana (FVTM), y (b) la Sierra Madre Oriental (SMO), abarcando la primera más del $90 \%$ del territorio del geoparque. Las rocas más antiguas en el área del GCM son de naturaleza sedimentaria; están expuestas exclusivamente en el área de la Sierra Baja y de forma dis- 
continua en la Barranca de Metztitlán. Se trata de una secuencia carbonatada del Cretácico Temprano y turbiditas calcáreas del Cretácico Tardío, que incluye, respectivamente, las formaciones El Doctor y Soyatal (cf. Mendoza-Rosales, 1990). Estas formaciones registran la evolución transgresiva de la margen occidental del Golfo de México, desde depósitos de carbonatos en un ambiente lagunar y de plataforma hasta depósitos de detritos carbonatados en una cuenca marina profunda (Wilson et al., 1955; Mendoza-Rosales, 1990), lo cual a su vez indica el inicio de un importante evento orogénico. Las rocas del Cretácico están significativamente acortadas con un estilo de "tectónica de piel delgada", característico del Cinturón Mexicano de Pliegues y Cabalgaduras (cf. Fitz-Díaz et al., 2011). Esta deformación se propagó de forma progresiva y episódica en el centro de México entre el Cretácico Tardío y el Paleógeno (Fitz-Díaz et al., 2014), y fue causada por la convergencia entre las placas Farallón y de Norteamérica; las principales estructuras en la zona son pliegues asimétricos y fallas inversas, todos con dirección de transporte dominante hacia el NE (Morán-Zenteno, 1994; Eguiluz et al., 2000; Salvador-Flores, 2001; Fitz-Díaz et al., 2011). El acortamiento horizontal de estas rocas causó levantamientos del orden de kilómetros, y es el principal responsable de la elevada topografía de la Sierra Madre Oriental y del GCM.
Durante el Oligoceno-Mioceno tardío, como producto del proceso de subducción de la Placa Farallón bajo la de Norteamérica, se formaron grandes volúmenes de rocas volcánicas calcoalcalinas, incluyendo (Geyne et al., 1963): (a) andesitas, dacitas y riolitas del Grupo Pachuca (Oligoceno), y (b) tobas riolíticas y riolitas de las formaciones Tezoantla y Cerezo (Mioceno), así como pórfidos riolíticos y dacíticos asociados. Estas rocas constituyen una de las manifestaciones más surorientales de la provincia volcánica de la Sierra Madre Occidental (SMOc) (e.g. Ferrari et al., 2005).

Durante el Plioceno-Holoceno la actividad ígnea ha obedecido a la subducción subhorizontal de la placa de Cocos por debajo de la Norteamericana, proceso que ha configurado la FVTM, individualizándola respecto al arco volcánico de la SMOc (Ferrari et al., 1999; Gómez-Tuena et al., 2005). La actividad reciente de la FVTM produjo en el área del GCM rocas volcánicas como (a) andesitas basálticas y depósitos de escoria de la Formación San Cristóbal (Plioceno; SGM, 2007), (b) la colada de los Prismas Basálticos (Fig. 1A), compuesta por basaltos con olivino (de edad pliocénica; fechados en $2.58 \pm 0.15$ Ma por Sánchez Rojas y Osorio Pérez, 2008), (c) riolitas con flujos de obsidiana de la unidad Las Navajas (Pleistoceno; SGM, 2007), (d) depósitos de lapilli y ceniza, ricos en pómez, de la Toba $\mathrm{Cu}$ bitos (Holoceno; SGM, 2007), y (e) la traquita de El Guajolote

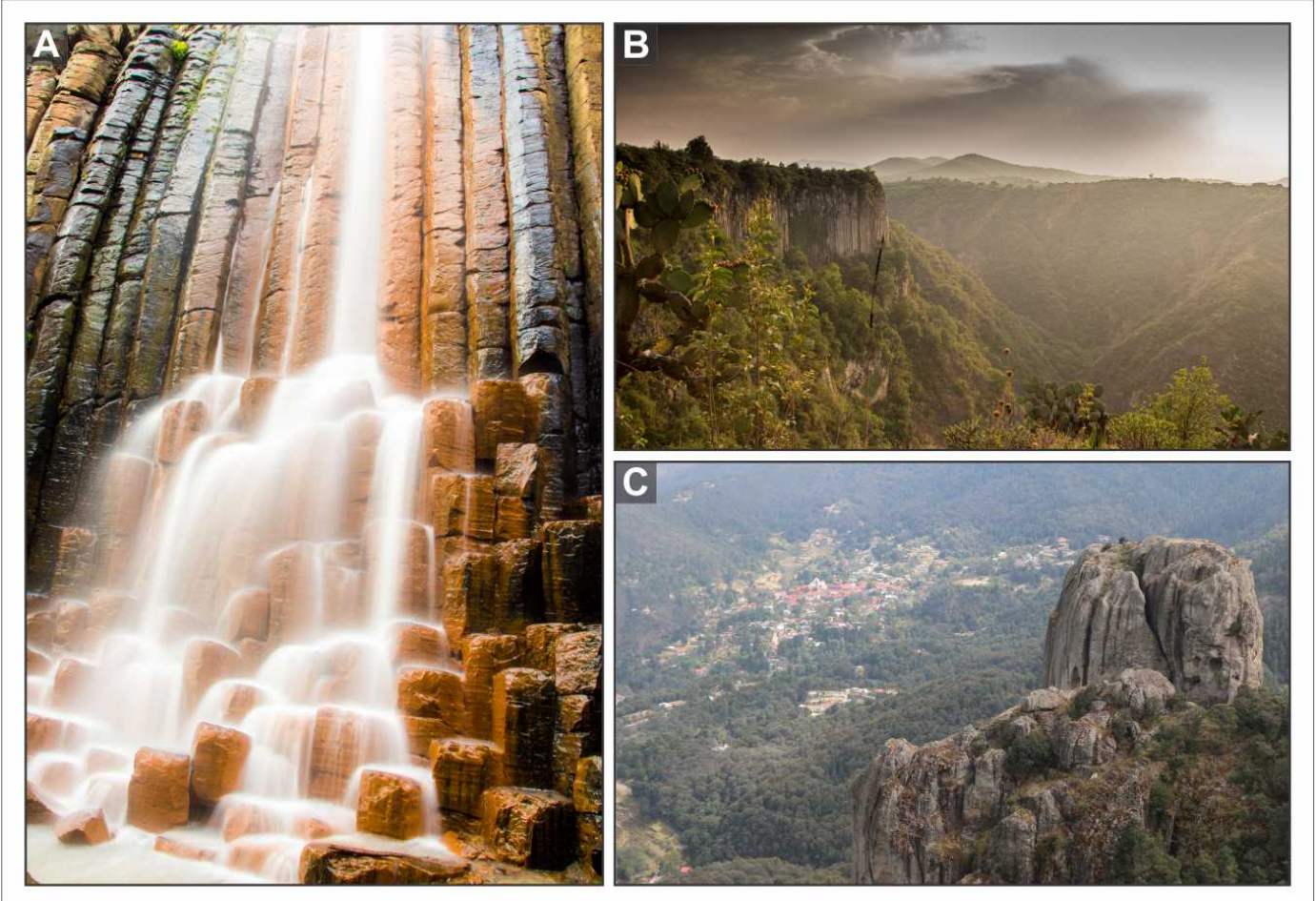

Figura 1: Tres geositios emblemáticos del Geoparque Mundial de la UNESCO Comarca Minera, Hidalgo (las ubicaciones se muestran en el mapa (ver Lámina 1) y las coordenadas en la Tabla 1). (A) Prismas basálticos de Santa María Regla y (B) Barranca de Aguacatitla, en el municipio de Huasca de Ocampo, y (C) Peñas de Las Monjas, en el municipio de Mineral del Chico.

Fig. 1. Three iconic geosites of the Comarca Minera, Hidalgo UNESCO Global Geopark (locations are shown in the map (see Plate 1) and coordinates provided in Table 1). (A) Columnar jointing of Santa María Regla and (B) Aguacatitla gorge, both in Huasca de Ocampo municipality, and (C) Las Monjas peaks, in Mineral del Chico municipality. 
(Holoceno; SGM, 1997).

El GCM comprende un distrito minero que se considera de clase mundial al tomarse en cuenta su producción metálica histórica; ésta alcanza una suma de $\sim 40 \times 10^{6} \mathrm{~kg}$ de plata (Geyne et al., 1963), extraída durante $\sim 500$ años de trabajos mineros que arrancaron a inicios de la Colonia Española (Probert, 1987) y cesaron paulatinamente durante la segunda mitad del siglo XX. En el distrito minero, conocido como distrito de Pachuca-Real del Monte, se explotó un sistema epitermal de baja sulfuración, de edad miocénica (relacionado con el magmatismo silícico de la SMOc), cuya mineralización se emplaza en vetas (Geyne et al., 1963; Dreier, 2005). Los minerales de importancia económica en el yacimiento son sulfuros, principalmente argentita y acantita, seguidos por sulfosales como la polibasita, la miargirita, la proustita y la stephanita, además de plata nativa (Geyne et al., 1963; McKee et al., 1992; Camprubí y Albinson, 2007).

\subsection{Geopatrimonio y geositios}

El territorio del GCM se caracteriza por un geopatrimonio sobresaliente, que justifica su inclusión en la Red Mundial de Geoparques e incluye los siguientes aspectos considerados de relevancia internacional: (a) el sistema epitermal ( $\mathrm{Ag}-\mathrm{Au})$ de Pachuca-Real del Monte, que fue uno de los más productivos del mundo entre los siglos XVI y XX; (b) la localidad tipo -en el Cerro San Cristóbal de Pachuca- de la tridimita y la cristobalita, minerales descubiertos por Gerhard vom Rath en 1868 y 1887, respectivamente (Sureda, 2008); (c) los basaltos con disyunción columnar de Huasca de Ocampo, conocidos popularmente como los "Prismas Basálticos de Santa María Regla", descritos y estudiados por Alexander von Humboldt en 1803 (cf. Holl, 1997) y considerados como los más largos del mundo; (d) el patrimonio industrial minero, que incluye haciendas de beneficio coloniales, así como obras mineras diversas, las más antiguas de las cuales son prehispánicas y explotaban la obsidiana (en la Sierra de las Navajas; Probert, 1987).

El GCM comprende una red de geositios cuyo propósito general es la geoconservación y el aprovechamiento turístico y educativo del geopatrimonio (Fig. 1). Esta red cuenta actualmente con 31 geositios catalogados, cuyas ubicaciones, temática (o principal interés; cf. Fuertes-Gutiérrez y Fernández-Martínez, 2010) y nombres se presentan en la Tabla 1.

La mayoría de los geositios se justifican por su interés geo-

Tabla 1: Listado de geositios del Geoparque Comarca Minera, Hidalgo (UTM Zona 14. WGS84)

Table 1: List of geosites of the Comarca Minera, Hidalgo Geopark (UTM Zona 14. WGS84)

\begin{tabular}{|c|c|c|c|c|c|c|}
\hline \# & Geositio & Principal interés & Provincia geológica & Coorden & das UTM & Municipio \\
\hline 1 & Prismas Basálticos & geología (vulcanología) & FVTM & 545602 & 2237490 & Huasca de Ocampo \\
\hline 4 & Depósitos tobáceos de Cubitos & geología (vulcanología) & FVTM & 527735 & 2222552 & Pachuca de Soto \\
\hline 5 & Obras mineras de El Milagro & geología / histórico-cultural & FVTM & 527532 & 2235850 & Mineral del Chico \\
\hline 6 & Cerro de las Navajas & geología / histórico-cultural & FVTM & 546679 & 2221034 & Epazoyucan \\
\hline 9 & Peña del Diablo-Piedra del Comal & geología (geomorfología) & FVTM & 536510 & 2230030 & Omitlán de Juárez \\
\hline 10 & Presa San Antonio & geología / histórico-cultural & FVTM & 545425 & 2237390 & Mineral del Monte \\
\hline 11 & Mirador Cerro del Lobo & geología (vulcanología) & FVTM & 528794 & 2225080 & Pachuca de Soto \\
\hline 12 & Peña del Aire (Mirador El León) & geología (geología regional) & FVTM-SMO & 550921 & 2240782 & Huasca de Ocampo \\
\hline 13 & Traquita El Guajolote & geología (petrología) & FVTM & 541883 & 2223119 & Epazoyucan \\
\hline 14 & Peñas Las Monjas & geología (geomorfología) & FVTM & 525532 & 2234710 & Mineral del Chico \\
\hline 20 & Mirador de Las Ventanas & geología (geomorfología) & FVTM & 527587 & 2232201 & Mineral del Chico \\
\hline 21 & Barranca de Aguacatitla & geología (estratigrafía) & FVTM-SMO & 547038 & 2239383 & Huasca de Ocampo \\
\hline 22 & Parque Estatal Bosque El Hiloche & biota-ecosistemas & FVTM & 533063 & 2227379 & Mineral del Monte \\
\hline 23 & $\begin{array}{c}\text { Reserva de la Biosfera Barranca de } \\
\text { Metztitlán }\end{array}$ & biota-ecosistemas & FVTM-SMO & 540645 & 2249000 & Atotonilco El Grande \\
\hline 24 & Cristo Rey & histórica-cultural & - & 529036 & 2225887 & Pachuca de Soto \\
\hline 25 & Reloj Monumental & histórica-cultural & - & 528035 & 2225614 & Pachuca de Soto \\
\hline 26 & Museo del Paste & histórica-cultural & - & 533002 & 2226895 & Mineral del Monte \\
\hline 27 & San Miguel Regla & histórica-cultural & - & 546311 & 2235610 & Huasca de Ocampo \\
\hline 28 & Ex-Hacienda La Purísima & histórica-cultural & - & 528534 & 2226498 & Pachuca de Soto \\
\hline 29 & Ex-Convento de San Andrés Apóstol & histórica-cultural & - & 538170 & 2213450 & Epazoyucan \\
\hline 30 & Panteón Inglés & histórica-cultural & - & 534803 & 2226785 & Mineral del Monte \\
\hline 31 & Ex-Convento de San Agustín & histórica-cultural & - & 534566 & 2243180 & Atotonilco El Grande \\
\hline
\end{tabular}

Abreviaturas / Abbreviations: FVTM. Faja Volcánica Transmexicana; SMO, Sierra Madre Oriental 


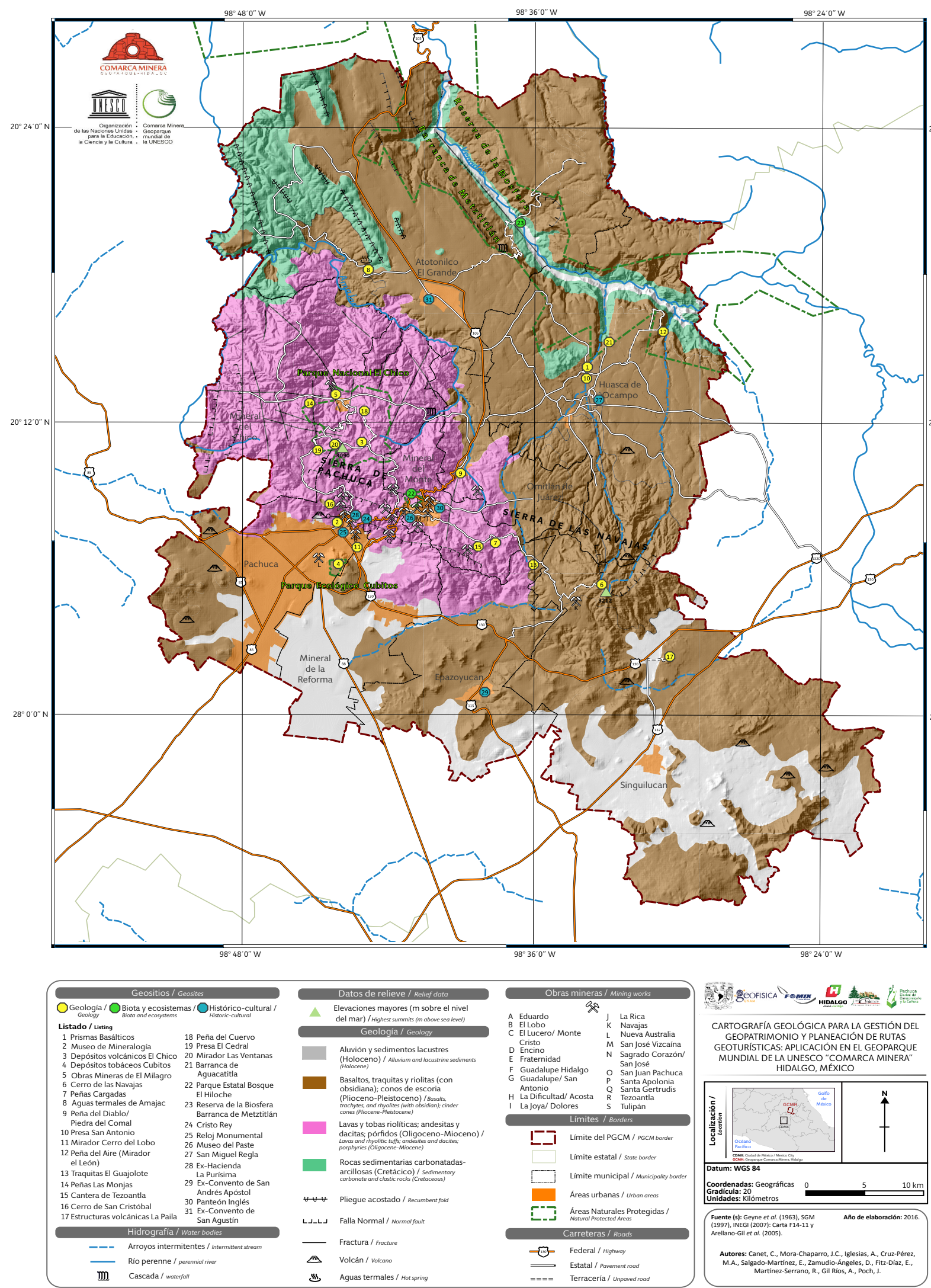

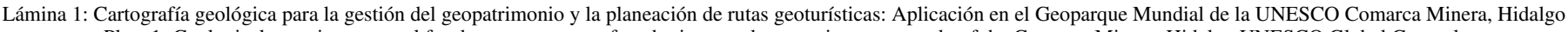
Plate 1: Geological mapping as a tool for the management of geoheritage and geotourism: case study of the Comarca Minera, Hidalgo UNESCO Global Geopark 
lógico (21 geositios), mientras que el restante incluye sitios cuyo interés son los elementos de la biota y los ecosistemas (2 geositios) o el patrimonio histórico-cultural (8 geositios). Los geositios de estas dos últimas categorías presentan siempre un vínculo con la geología o con el legado minero, y la mayoría de geositios geológicos tienen asimismo un interés históricocultural. Los geositios son lugares a los que les atribuimos un valor científico y didáctico; muchos de ellos son gestionados por ejidos (12 geositios) y por instituciones gubernamentales a distintos niveles (13 geositios) o privadas (5 geositios), y únicamente un geositio carece de gestión regular.

El nombre de los geositios se asignó de acuerdo con la toponimia original, tal y como es utilizada por los pobladores locales (e.g. Peñas Cargadas, Peña del Aire, Las Monjas, La Paila); en el caso de edificios administrados por instituciones públicas $\mathrm{o}$ asociaciones civiles se aplican los correspondientes nombres registrados (e.g. Museo de Mineralogía, Museo del Paste). Se han establecido cuatro georrutas, que agrupan geositios con una temática y un discurso turístico coherentes: (a) Ruta Humboldt, (b) Ruta Histórico-Minera, (c) Ruta Geo-cultural, y (d) Ruta Geo-natural.

\section{Metodología}

Para la representación 3D del relieve en el mapa base se utilizaron datos vectoriales proveídos por INEGI (2007), en su Carta Topográfica F14-11, escala 1:250,000, la cual es también la fuente para las vías de acceso pavimentadas (autopistas y carreteras). Por otro lado, algunos caminos de terracería que dan acceso a geositios fueron cartografiados en campo. Para la hidrografía se usaron los metadatos geográficos de MadereyR. y Torres-Ruata (1990), disponibles en los catálogos de la CONABIO (http://www.conabio.gob.mx). Los polígonos de las áreas naturales protegidas federales (Parque Nacional El Chico y Reserva de la Biosfera Barranca de Metztitlán) se obtuvieron de CONANP (2010, 2012). La geología se basa en Geyne et al. (1963) y en las cartas geológico-mineras de Pachuca F1411, escala 1:250,000 (SGM, 1997) y F14-D8, escala 1:50,000 (SGM, 2007).

\section{Conclusiones}

El GCM contiene una red de 31 geositios que permiten dar a conocer un geopatrimonio excepcional, cuyo eje temático es la minería de la plata en el distrito minero de Pachuca-Real del Monte. La elevada geodiversidad del GCM, apreciable en sus geositios, se debe a que en el área convergen dos provincias fisiográficas (SMO y FVTM), así como dos provincias magmáticas (FVTM y SMOc). La cartografía geológica simplificada se integra en el mapa geoturístico del GCM presentado en este trabajo, lo que permite visualizar las provincias antes mencionadas y asociarles geositios para trazar georrutas con un discurso turístico, didáctico y de conservación coherente con el marco y la historia geológicos.

\section{Software}

Se utilizó QGIS Desktop 2.12.2 Lyon y Adobe Illustrator CC. Para la conversión de coordenadas se utilizó Geo Tracker 3.3.0.1338 para Android.

\section{Agradecimientos}

El financiamiento para esta investigación proviene de los proyectos Fondo Mixto CONACYT-Gobierno del Estado de Hidalgo (267903) y PAPIME, DGAPA-UNAM (PE107617). Los autores agradecen los valiosos comentarios y correcciones realizados a este trabajo por José Luis Palacio Prieto, Penélope López Quiroz, Luca Ferrari, Javier Osorno y Enrique Cabral Cano. Por su colaboración y valiosas aportaciones, nuestro agradecimiento va a las siguientes personas: Omar Ramírez García, Adrián Rivera González, Rogelio Hernández Vergara, Berenice Peláez Pavón, Luis Abimael Granados Lugo, Lucero Morelos Rodríguez, Paulette Escalona Muñoz, Eduardo García Alonso, Gabriela Y. Castañón, Màrius Ramírez Cardona, Enrique Cruz Chávez, Jesica Salas, Rosa María Prol Ledesma, Miguel Flores, Ana Enciso Vargas y César I. Aldama Muciño. Agradecemos por su valiosa cooperación y facilidades de acceso a los ejidos de Matías Rodríguez, Nopalillo, San Juan Tizahuapan, San Sebastián, San Miguel Regla, Santa María Amajac, Santa María Regla, Puentecillas y Xoloxtitla, así como a las organizaciones civiles del Centro Turístico Barranca de Aguacatitla A.C., Museo del Paste Mineral del Monte A.C., y Ecoparque Aventuras Palaguth. Agradecemos a la Reserva de la Biosfera Barranca de Metztitlán, así como a Efrén Cortés, Sergio Gómez y Alejandro Zamperio, guías certificados, por su apoyo en el trabajo de campo. También gracias por las fotografías a Daniel Acosta, Iván Razo, Rodrigo Ruiz y María Elena Santos, y por el apoyo en diseño gráfico a Jacqueline Cisneros y Anaid Galicia.

\section{Referencias}

Baker, K. M., Petcovic, H., Wisniewska, M., Libarkin, J., 2012. Spatial Signatures of Mapping Expertise Among Field Geologists. Cartography and Geographic Information Science 39 (3), 119-132, doi: $10.1559 / 15230406393119$

Camprubí, A., Albinson, T., 2007. Epithermal deposits in México-Update of current knowledge, and an empirical reclassification. Geological Society of America Special Paper 422, 377-415Doi: 10.1130/2007.2422(14).

Carcavilla, L., López-Martínez, J., Durán, J. J., 2007. Patrimonio geológico y geodiversidad: investigación, conservación, gestión y relación con los espacios naturales protegidos. No. 7 en Serie Cuadernos del Museo Geominero. Instituto Geológico y Minero de España, Madrid, 360 pp.

Colorado Geological Survey (CGS), 2016. Geologic Mapping (en línea). CGS, disponible en <http://coloradogeologicalsurvey.org/geologicmapping/>, consultado en diciembre de 2016.

Comisión Nacional de Áreas Naturales Protegidas (CONANP), 2010. Reservas de la Biosfera (en línea). CONANP, disponible en $<$ http://www.conanp.gob.mx/que_hacemos/reservas_biosfera.php $>$, consultado en diciembre de 2016.

Comisión Nacional de Áreas Naturales Protegidas (CONANP), 2012. Parques Nacionales (en línea). CONANP, disponible en $<$ http://www.conanp.gob.mx/que_hacemos/parques_nacionales.php $>$, consultado en diciembre de 2016. 
Dreier, J. E., 2005. The Environment of Vein Formation and Ore Deposition in the Purisima-Colon Vein System, Pachuca Real del Monte District, Hidalgo, Mexico. Economic Geology and the Bulletin of the Society of Economic Geologists 100 (7), 1325-1347, doi: 10.2113/gsecongeo.100.7.1325.

Eguiluz De Antuñano, S., Aranda García, M., Marrett, R., 2000. Tectónica de la Sierra Madre Oriental, México. Boletín de la Sociedad Geológica Mexicana 53, 1-26.

Ferrari, L., López-Martínez, M., Aguirre-Díaz, G., Carrasco-Núñez, G., 1999. Space-time patterns of Cenozoic arc volcanism in central Mexico: From the Sierra Madre Occidental to the Mexican Volcanic Belt. Geology 27 (4), 303306, doi: 10.1130/0091-7613(1999)027<0303:STPOCA >2.3.CO;2.

Ferrari, L., Valencia-Moreno, M., Bryan, S., 2005. Magmatismo y tectónica en la Sierra Madre Occidental y su relación con la evolución de la margen occidental de Norteamérica. Boletín de la Sociedad Geológica Mexicana, Volumen Conmemorativo del Centenario, Temas Selectos de la Geología Mexicana 57 (3), 343-378, 10.2307/24920394.

Fitz-Diaz, E., Hudleston, P., Tolson, G., 2011. Comparison of tectonic styles in the Mexican and Canadian Rocky Mountain Fold-Thrust Belt, en Poblet, J., Lisle, R. (Eds.), Kinematics and Tectonic Styles of Fold-Thrust Belt. Geological Society, London, Special Publications 349, 149-167, 10.1144/SP349.8.

Fitz-Diaz, E., Hudleston, P., Tolson, G., Pluijm, B. v. d., 2014. Progressive, episodic deformation in the Mexican Fold-Thrust Belt (central Mexico): evidence from isotopic dating of folds and faults. International Geology Review 56 (6), 734-755, 10.1080/00206814.2014.896228.

Fuertes-Gutiérrez, I., Fernández-Martínez, E., 2010. Geosites Inventory in the Leon Province (Northwestern Spain): A Tool to Introduce Geoheritage into Regional Environmental Management. Geoheritage 2, 57-75, 10.1007/s12371-010-0012-y.

Geyne, A.R., Fries, Jr. C., Segerstrom, K., Black, R.F., Wilson, I.F., 1963. Geología y yacimientos minerales del Distrito de Pachuca-Real del Monte, Estado de Hidalgo, México. Consejo de Recursos Naturales no Renovables, Publicación 5 E223 pp.

Global Geoparks Network (GGN), 2017. Global Geoparks Network (en línea). GGN, disponible en $<$ http://www.globalgeopark.org $>$, consultado en mayo de 2017.

Gray, M., 2004. Geodiversity: Valuing and Conserving Abiotic Nature. 2nd Edition, Wiley-Blackwell, 508 pp.

Greiving, S., Ubaura, M., Tesliar, J., 2016. Spatial Planning and Resilience Following Disasters: International and Comparative Perspectives. Bristol, Reino Unido, Policy Press, 360.

Gómez-Tuena, A., Orozco-Esquivel, M. T., Ferrari, L., 2005. Petrogénesis ígnea de la Faja Volcánica Transmexicana. Boletín de la Sociedad Geológica Mexicana 57 (3), 227-283, 10.2307/24920391.

Holl, F., 1997. Alejandro de Humboldt en México. Secretaría de Hacienda y Crédito Público, Instituto Nacional de Antropología e Historia, Instituto Goethe, México, 217 pp.

Hose, T. A., 2016. Three centuries (1670-1970) of appreciating physical landscapes. Geological Society, London, Special Publications 417 (1), 1-23, 10.1144/SP417.15.

Hose, T. A., Vasiljević, D. A., 2012. Defining the Nature and Purpose of Modern Geotourism with Particular Reference to the United Kingdom and South-East Europe. Geoheritage 4 (1-2), 25-43, 10.1007/s12371-011-00500.

Hose, T.A., 1995. Selling the story of Britain's stone. Environmental Interpretation $10(2), 16-17$.

Instituto Geológico y Minero de España (IGME), 2016. Cartografía del IGME, (en línea). IGME, disponible en <http://info.igme.es/cartografiadigital/>, consultado en diciembre de 2016.

Instituto Nacional de Estadística y Geografía (INEGI), 2007. Carta Topográfica F14-11, Hoja Pachuca de Soto, Escala 1:250,000. INEGI.

Instituto Nacional de Estadística y Geografía (INEGI), 2013. Anuario estadís- tico y geográfico de Hidalgo, México. INEGI, 583 p.

Inventario Español de Lugares de Interés Geológico (IELIG), 2016. Disponible en $<$ http://info.igme.es/ielig $>$, consultado en diciembre de 2016.

IUGS-Geoheritage Task Group (GTG), 2016. Disponible en $<$ http://geoheritage-iugs.mnhn.fr>, consultado en diciembre de 2016.

Maderey-R., L.E., Torres-Ruata, C., 1990. Hidrografía e hidrometría, IV.6.1 (A). Atlas Nacional del México, Vol. II, Escala 1:4,000,000. Instituto de Geografía, UNAM.

McKee, E. H., Dreier, J. E., Noble, D. C., 1992. Early Miocene hydrothermal activity at Pachuca-Real del Monte, Mexico; an example of space-time association of volcanism and epithermal Ag-Au vein mineralization. Economic Geology 87 (6), 1635-1637, 10.2113/gsecongeo.87.6.1635.

Mendoza-Rosales, C.C., 1990. Estructura del sector Saja (Cabalgadura El Doctor) estados de Hidalgo y Querétaro. Facultad de Ingeniería, UNAM, Tesis Profesional, $64 \mathrm{pp}$.

Morán-Zenteno, D., 1994. The Geology of the Mexican Republic. American Association of Petroleum Geologists. Studies in Geology 39, 160.

Palacio Prieto, J. L., 2013a. Geositios, geomorfositios y geoparques: importancia, situación actual y perspectivas en México. Investigaciones Geográficas, Boletín del Instituto de Geografía 82, 24-37, 10.14350/rig.32817.

Palacio Prieto, J. L., 2013b. Sin protección legal, el patrimonio geológico y geomorfológico de México. Boletín UNAM-DGCS-450.

Probert, A., 1987. En pos de la plata. Episodios Mineros en la Historia Hidalguense. Pachuca, Hidalgo, Colección Hidalguense, Dirección General de Publicaciones e Impresos del Gobierno del Estado de Hidalgo, 446 pp.

Prosser, C. D., Burek, C. V., Evans, D. H., Gordon, J. E., Kirkbride, V. B., Rennie, A. F., Walmsley, C. A., 2010. Conserving Geodiversity Sites in a Changing Climate: Management Challenges and Responses. Geoheritage 2 (3), 123-136, 10.1007/s12371-010-0016-7.

Raisz, E., 1959. Landforms of Mexico: escala ca. 1:3,000,000. Cambridge, Massachussetts, U.S. Office of Naval Research, Geography Branch.

Salvador-Flores, R., 2001. Origen sedimentológico y estratigrafía del Paleolago de Amajac, Hidalgo. Universidad Nacional Autónoma de México, Facultad de Ingeniería, México, D. F., tesis profesional, inédita, 87 p.

Servicio Geológico Mexicano (SGM), 1997. Carta Geológico-Minera Pachuca F14-11, 1:250,000. SGM, Pachuca, Hidalgo, México.

Servicio Geológico Mexicano (SGM), 2007. Carta Geológico-Minera Pachuca F14-D8, 1:50,000. SGM, Pachuca, Hidalgo, México.

Soller, D.R., 2004. Introduction to Geologic Mapping. McGraw-Hill Yearbook of Science \& Technology, 128-130.

Sureda, R.J., 2008. Historia de la Mineralogía. Serie Correlación Geológica, 23 (ISSN on-line 1666- 9479). Universidad Nacional de Tucumán, Argentina, $189 \mathrm{pp}$.

Sveriges geologiska undersökning (SGU), 2016. Tävlingen Geologiskt Arv (Concurso de Patrimonio Geológico, en sueco). Disponible en $\quad<\mathrm{http}: / / \mathrm{www} . s g u . s e /$ evenemang/genomforda-evenemang/tavlingengeologiskt-arv $>$, consultado en diciembre de 2016.

Sánchez Rojas, E., Osorio Pérez, M., 2008. Geología y petrogénesis de los Prismas Basálticos. Santa María Regla, Hgo. GeoCiencia, Revista del Servicio Geológico Mexicano 3, 5-24.

UNESCO Global Geoparks, 2017. Disponible $<$ http://www.unesco.org/new/en/natural-sciences/environment/earthsciences/unesco-global-geoparks $>$, consultado en mayo de 2017.

Wilson, B.W., Hernández, J.P., Meave, E., 1955. Un banco calizo del Cretácico en la parte oriental del estado de Querétaro, México. Boletín de la Sociedad Geológica Mexicana 18, 1-10.

This article accompanies the following material:

HTML: $\quad$ DOI: 10.22201/igg.terradigitalis.2017.2.21.72

Interactive map: DOI: 10.22201/igg.terradigitalis.2017.2.21.74

Static map: $\quad$ DOI: 10.22201/igg.terradigitalis.2017.2.21.73 